\title{
Intelligent Architecture: Embedding Spaces with a Mind for Augmented Interaction
}

\author{
Flavia Sparacino \\ Sensing Places and MIT \\ flavia@sensingplaces.com \\ flavia@media.mit.edu
}

\begin{abstract}
Our society's modalities of communication are rapidly changing: we divide our activities between real and digital worlds and our daily lives are characterized by our constant access-to and processing-of a vast quantity and variety of information. These transformations of our lifestyle demand both a new architecture and interaction modalities that support the new as well as old ways of communicating and living.
\end{abstract}

As a consequence of the prevalent role of information in today's society, architecture is presently at a turning point. Screens are everywhere, from the billboards which dot the contemporary urban cityscape, to the video walls which welcome us in the entry-halls of corporate headquarter buildings, to our desktop computer monitor at home, the PDA in our pocket, or the tiny privateeye screens of wearable computers. Wearable computers are starting to transform our technological landscape by reshaping the heavy, bulky desktop computer into a lightweight, portable device that's accessible to people at any time. Computation and sensing are moving from computers and devices into the environment itself. The space around us is instrumented with sensors and displays, and this tends to reflect a widespead need to blend together the information space with our physical space. "Augmented reality" and "mixed reality" are the terms most often used to refer to this type of media-enhanced interactive space. The combination of large public and miniature personal digital displays together with distributed computing and sensing intelligence offers unprecedented opportunities to merge the virtual and the real, the information landscape of the Internet with the urban landscape of the city, to transform digital animated media in public installations, in storytellers, also by means of personal wearable technology.

To meet the challenges of the new information- and technology-inspired architecture we need to think of the architectural space not simply as a container but as a living body endowed with sensors, actuators, and a brain (a mind), a space capable of assisting people in the course of their activities within such spaces.

On the basis of my work and research I will argue that intelligent architecture needs to be supported by three forms of intelligence: perceptual intelligence, which captures people's presence and movement in the space in a natural and non-encumbering way; interpretive intelligence, which "understands" people's actions and is capable of making informed guesses about their behavior; and narrative intelligence, which presents us with information, 
articulated stories, images, and animations, in the right place, at the right time, all tailored to our needs and preferences.

This talk will describe and illustrate a series of models, technological platforms and installations the author developed originally at the MIT Media Lab (1994 to 2002) and later commercially for Sensing Places (2003 to 2005). They contribute to defining new trends in architecture that merge virtual and real spaces, and are currently in the process of reshaping the way we live and experience the museum, the home, the theater, and the modern city. 